\title{
Vehicular Networks Based Intelligent Transportation System
}

\author{
Stephen Neal Joshua. Eali ${ }^{1}$, N. Thirupathi Rao $^{2}$ and Debnath Bhattacharyya ${ }^{3}$ \\ Dept. of Computer Science and Engineering, Vignan's Institute of Information \\ Technology, Visakhapatnam, AP, India \\ 'nakkathiru@gmail.com,1402,3debnathb@gmail.com
}

\begin{abstract}
In this paper we tend to propose the instrument for recognizing fast vehicle in transport unintended Network (VANET). Keeping in mind the tip goal to understand the surrounding condition, each vehicle often communicates its position, temporal order knowledge in its correspondence run. The Central Server registers the traditional speed, blockage and street mischances of the vehicle utilizing time and position knowledge gotten from the RSUs. At the purpose once a vehicle is found to own a lot of speed or Road mishap had happened than the specific space, the Central Server communicates this knowledge to any or all RSUs in its vary. At the purpose once the vehicle once more comes into the scope of a RSU, it illuminates the vehicle to drive within as way as doable or to require redirection utilizing a notice message. Keeping in mind the tip goal to assess the execution of our set up, we've got utilised Veins 0.5 breed check system that chips away at OMNeT++ as a system check system, Simulation of Urban Movableness (SUMO) as traffic check system.
\end{abstract}

Keywords: Roads, Traffic, Lights, MANETs, VANETs, Robust methods, Simulation models, Check system, Road aspect unit

\section{Introduction}

Traffic blockage on streets is a prime issue in city communities. The clog and car collecting trouble is joined through steady chance mischances. Absence of avenue traffic safety and increment in the number of speedy automobile takes a no of valuable human lives and represents a proper risk to our condition additionally [1][2]. One-of-a-kind consequences are recognized with natural infection and energy squander. As indicated through countrywide dual carriageway Traffic safety administration, 6.3 million Police discovered traffic mishaps, and forty three people were slaughtered. The financial system ejects induced due to these mischance had been greater than $\$ 230$ billion and millions of individuals were harmed. Preparatory protection measures like airbags and safety belts are applied yet they cannot wipe out troubles because of motive force's powerlessness to foresee the circumstance early. On an interstate or in a defining moment a vehicle cannot foresee the prevailing pace of various cars [3][4][5].

In any case, with the utilization of remote correspondence hardware, sensor and pc velocity may be expected and be aware message sent at everyday periods ought to constrain the danger of capability mishaps. A Vehicular advert-Hoc community, or VANET, is a type of cell specifically appointed gadget which gives interchanges among cars, amongst near-by way of motors, and adjacent fixed gadgets, normally depicted as a roadside unit (RSU). The

Article history:

Received (April 3, 2019), Review Result (July 24, 2019), Accepted (August 29, 2019) 
fundamental goal of VANET is to present safety and solace to guests, drivers and other road customers [6][7]. To perform this extremely good electronic system can be associated with each automobile that allows you to deliver advert Hoc community availability to the guests. each automobile is supplied with VANET device, could be a hub inside the ad-Hoc set up and might get and hand-off others messages however the machine. Road sign signals, Collision cautioning and traffic view will give the motive force to choose the best manner en route to attain the purpose. There is moreover one-of-a-kind administrations like combined media and internet availability workplaces for travelers, all of with the intention to receive inside the faraway scope territory of every vehicle [8][9].

The circumstance deteriorates if there may be a mishap along one manner or a major truck containing merchandise has had a breakdown at the smaller crossing factor. Lengthy lines, slight twisting lines of shifting vehicle coincidence and the situation can be incredibly demoralizing for one to make use of sure infamous streets. Driving via a town where there are smaller streets and poor preventing zone amid choose hours is a disaster for enables and the fretful. Those shape of such encounters and perceptions that shrewd Transportation systems (ITS) can end up surprisingly convenient in facilitating, if now not settling this traffic wasteland franticness.

\section{Literature review}

Vehicular Communication frameworks are very nearly down to earth arrangement. Regardless, their security and security assurance is one of the issues that have been tended to. With a specific end goal to demonstrate the attainability of secure $\mathrm{VC}$, certain executions are required [1].

The fundamental goal of these venture and research are to create conventions and apparatuses to give satisfactory remote correspondence between vehicles. As, we as a whole realize that these examinations and practices are still re-enactment based on the grounds that it's infeasible to utilize genuine vehicles for the huge situation in various conditions to grow new calculations and convention for VANET [2].

The goal clarified in this paper was to sidestep the requirement for costly foundation: their real downside in the relatively complex systems administration conventions and the requirement for their noteworthy infiltration before their application can ended up noticeably viable [3].

This paper examines on the innovation trial that was directed utilizing $5.9 \mathrm{GHz}$ Dedicated Short range Communication (DSRC) recurrence and convention for Vehicle-to-foundation (V2I) and Vehicle-to-Vehicle (V2V) Applications in Singapore [4].

This paper proposes a New Automatic fall back calculation that can separate the two sorts of misfortunes and hone the precision of the rate adjustment prepare [6][7] VANET (vehicular Transportation system) is an open standard for bury vehicular correspondence in a street or with street side station with the vehicles. In this way directing in VANET is utilized to give security administrations. However, these conventions confront a few issues for the most part flooding of bundles arrange overhead and way misfortune that keep organize from ensuring their required high parcel conveyance proportion and low postponement.

Vehicular Ad hoc Networks is a sort of unique wireless specially appointed system, which has the qualities of high hub versatility and fast topology changes. VANET gives a distinguished way to deal with Intelligent Transport System (ITS) [8][10][11]. The Vehicular Networks can provide wide assortment of services, go from safety related cautioning frameworks to enhance $\mathrm{d}$ route instruments and additionally data and entertainment 
applications. These extra components make the routing and different administrations all the more difficult and cause vulner capacity in network administrations. These issues incorporate network k design, vanet conventions, steering calculations, and also security issues. In this paper, we give an audit to the looks into identified with existing and rising directing protocol in VANET [11][12].

Inter Vehicle Communication (IVC) Protocol can possibly build the security, efficiency, and comfort of transportation frameworks including planes, trains, cars, and robots [9]. The applications focused on incorporate shared systems for web surfing, facilitated braking, runway invasion anticipation, versatile traffic control, vehicle arrangements, and numerous others. The differences of the applications and their potential correspondence conventions have tested a deliberate writing review. We apply a classification system to IVC applications to give a scientific categorization to point by point investigation of their correspondence necessities [13]. The applications are separated into sort classes which share basic correspondence association and execution necessities. IVC conventions are reviewed independently and their principal qualities are uncovered. The convention qualities are then used to decide the importance of specific conventions to specific sorts of IVC applications.

To offer some benefit included administrations like email, sound/video sharing and so on., Various correspondences sorts are Vehicle to Vehicle (V2V), Vehicle to Infrastructure (V2I), Vehicle to Roadside (V2R), Hybrid Models, for example, Vehicle to Vehicle (V2V) and Vehicle to Infrastructure (V2I) and Vehicle to Vehicle (V2V) and Vehicle to Roadside (V2R). Vehicle to Vehicle correspondence approach is most suited for short range vehicular systems. Interchanges amongst vehicles and RSUs are upheld by Vehicle to-Infrastructure (V2I) convention and Vehicle-to-Roadside (V2R) convention.

\section{Simulation methodology}

The Purpose of our Study is to research the simulation of conveyance networks in realistic situation for that we decide the Andhra University close region for Simulation. Previous Studies on VANETS [1] centered a lot of on image implementation. In Singapore that they had train run with five. $6 \mathrm{GHz}$ DSRC that shown few positive results. As Implementation of VANETSs on check beds would value a lot of. We have a tendency to square measure restricted towards the pure Simulation with none image implementation. We have a tendency to centered on Single surroundings of VANETs i.e.., in town to gauge the performance of various routing protocols like AODV, DSR, DSDV, OLSR and DYMO on these lines in our investigation we have a tendency to contemplate distinctive sensible things comprise of Andhra faculty RSUOBU scenario to form fitting things to the recreation assessment of VANETs [10][11].

Tool choice is vital to search out results and finding in an exceedingly a lot of correct manner. We've got hand-picked OMNeT++ 4.6 to validate our findings. Rather than containing express and hardwired support for $\mathrm{pc}$ networks or alternative areas, it provides the infrastructure for writing such simulations. Specific application square measures are catered by numerous simulation models and frameworks, most of them square measure open supply.

\section{Simulation setup}

The first step for simulation setup is to create Vehicular Ad Hoc Networks. Network is created by using Create Project that is provided by OMNeT++. For Simulation of setup for VANETs we followed following mentioned steps:

\subsection{Sumo}


Sumo is likewise outstanding with other name i.e.., Traffic Simulator. In VANETs Node Moments were appeared in Traffic Simulator and Packets Delivering, Routing Issues were considered in Network Simulator OMNeT++. All the while these two were associated with Proxy server called as Tracl Manager. In the first place we have to Import our nearby guide from www.openstreetmap.org in the wake of bringing in it we have to utilize netconvert, polyconvert, randomTrips.py and Duroutor summons from SUMO-0.22.0 CMD Prompt.

\subsection{Netconvert}

Netconvert imports computerized street systems from various sources and creates street organizes that can be utilized yet different apparatuses from the bundle Purpose: Road Networks imports and change netconvert -osm-documents vizag.osm -o vizag.net.xml

\subsection{Polyconvert}

Polyconvert imports geometrical shapes (polygons or purposes of enthusiasm) from various sources, changes over them to a portrayal that might be pictured utilizing SUMO-GUI. Reason: Polygon and POI import, transformation, and projection Polyconvert -net-document vizag.net.xml -osm-records vizag.osm -typefile typemap.xml -o vizag.poly.xml.

sumo-0.22.0\tools $\backslash$ trip \randomTrips.py $-n$ vizag.net.xml $-r$ vizag.rou.xml $-\mathbf{e} 50-\boldsymbol{l}$

\section{Edit properties}

In this progression we allotted distinctive esteems in every parameter of vehicular hubs and designed every parameter. Other than these hub settings other essential settings for reproduce and correspondence among different hubs with the end goal that characterizing the aggregate reproduction time, flag determination, correspondence sort and so forth.

Table 1. Parameters taken for reenactment SUMO reproduction parameters for Vehicles and OMNET++

\begin{tabular}{|c|c|c|c|}
\hline Parameter (SUMO) & Values & Parameters Used (OMNeT++) & value \\
\hline Maximum vehicle speed & $33 \mathrm{~m} / \mathrm{sec}$ & sim-time-limit & $7000 \mathrm{~s}$ \\
\hline Maximum accelerati on & $2.6 \mathrm{~m} / \mathrm{s} 2$ & Mac.queuelength & 8 \\
\hline Maximum decelerati on & $4.5 \mathrm{~m} / \mathrm{s} 2$ & Mac.maxTxAttempt s & 12 \\
\hline vehicle length & $5 \mathrm{~m}$ & Mac.bitrate & $10 \mathrm{Mbps}$ \\
\hline vehicle width & $3.5 \mathrm{~m}$ & Mac.txpower & $10 \mathrm{~mW}$ \\
\hline Driver imperfection & 0.5 & Mac.contentionWin dow & 200 \\
\hline- & - & Mac.slotduration & $0.09 \mathrm{sec}$ \\
\hline- & - & Phy.sensitivity & $60 \mathrm{dBM}$ \\
\hline- & - & UpdateInterval & $0.5 \mathrm{sec}$ \\
\hline
\end{tabular}

In this reenactment examine for the RSU-OBU situation, we have chosen the accompanying system and Traffic parameters and devices for vehicular hubs and correspondence among them as appeared in [Table 1].

\section{Simulation scenario}


In order to carry out the performance of the considered model, the simulation model had selected and the simulation scenario had shown as an example in the below [Figure 1]. The software considered for the current simulation model was the SUMO software. The movement of vehicles on the four road junctions and other cases of road junctions and combinations of roads are considered as various scenarios and an example of one of the scenario model had shown in the current figure. The initiation and the mode of working mechanism of the current scenario can be observed as follows.

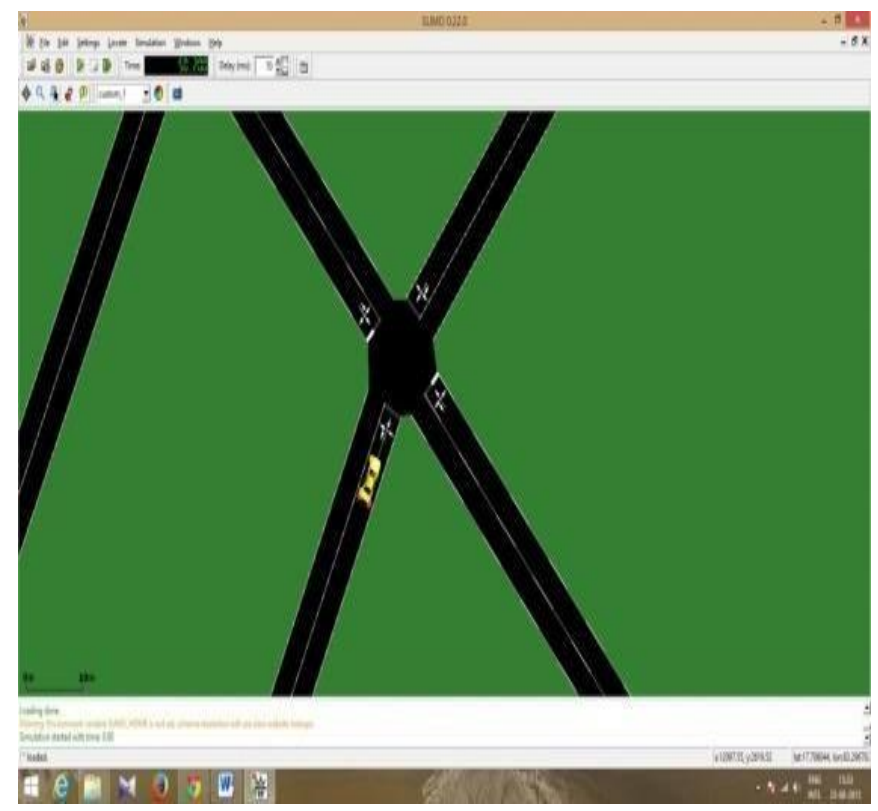

Figure 1. Showing node moment on SUMO (simulation of urban mobility)

\section{Results and analysis}

For this Simulation, we have worked with essentially, a situation to check whether we can surmised an adequate separation between the RSU, regarding usable postpone times for the messages. For this situation we have utilized both intermittent information and burst data. The RSUs are set at $100 \mathrm{~m}$, from the beginning stage, so we get a base estimation for the deferral presented by the line. Parameters: Road - 2400m long, RSU 1 position - 100m from beginning stage, RSU2 position - $1100 \mathrm{~m}$ from begin point. Our supposition for this situation is that the postponement in sending the messages is inconsequential, with values under second. In the wake of examining the outcomes (grouping outline, vector information, line time graph), we can see that the holding up time is of course, exceptionally small. We can likewise watch, for correlation purposes, the line length diagram, to perceive how it responds as the messages are being made, put away and sent to and from the line. In the last piece of the reproduction, the line length increments bit by bit, in light of the fact that the OBU moves out of range from the second RSU and doesn't send any information any more [Figure 2]. The spikes on the outline speak to the burst information that is made and sent. In this situation, we have a separation between the 20 second check and the 32 second, amid which the auto moves out of range from the primary RSU, and in scope of the second. This is noticeable on the succession graph, as there are no reference points being gotten by the OBU amind this period. 

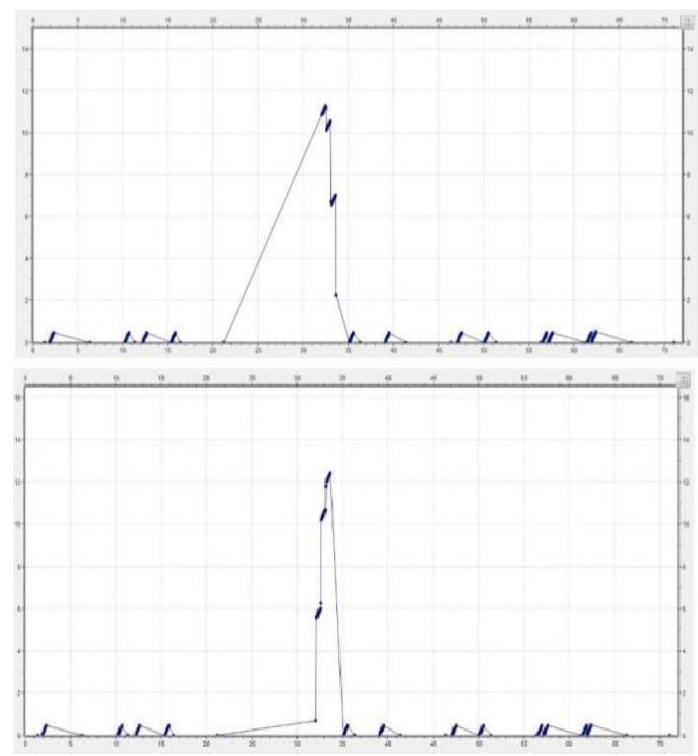

Figure 2. Queuing time (in seconds)
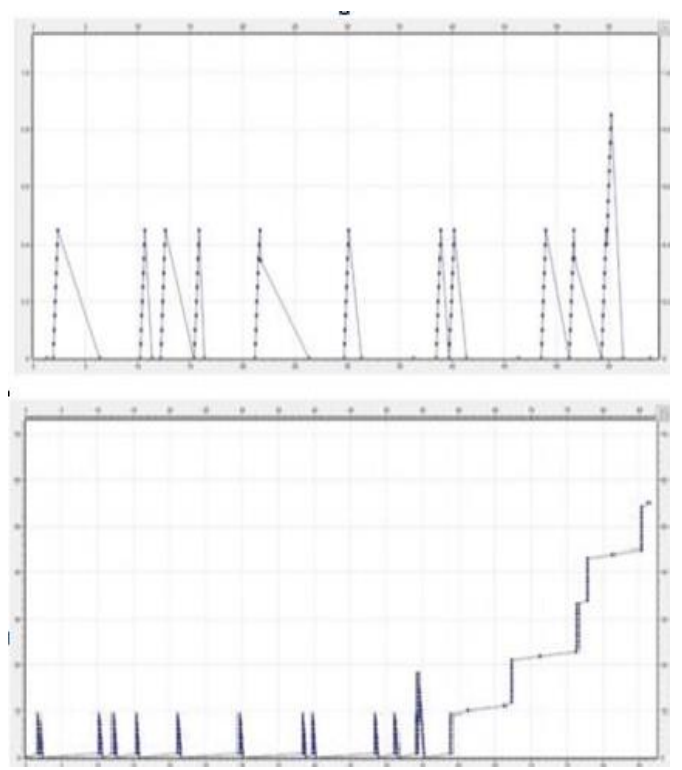

Figure 3. Queue length vector

In the event that we investigate the Queuing time graph [Figure 3], we can see the lining time expanding while the separation happens. At the point when the OBU is in run once more, the esteem diminishes, as the information is being sent. We can watch that the line time esteem increments more than 10 seconds, which corresponds with the timeframe in which the OBU is not in scope of the RSU. As should be obvious, there is a distinction in the lining time chart between the LIFO and the FIFO line, which is not out of the ordinary, given the way that each of these lines work. In the event that on account of the FIFO line, the expansion in the deferral is straight, in light of the fact that the messages put away in it need to sit tight for longer to be sent, the LIFO line, given that it sends the last messages to begin with, will have an unexpected increment in the lining time. The most extreme esteem that this postponement comes to is the 
same in both cases. If we analyze the Queue length outline from the past situation [Figure 4] with the one we have here [Figure 5], we can see the line length expanding as the OBU is moving out of range from the primary RSU and in scope of the second.

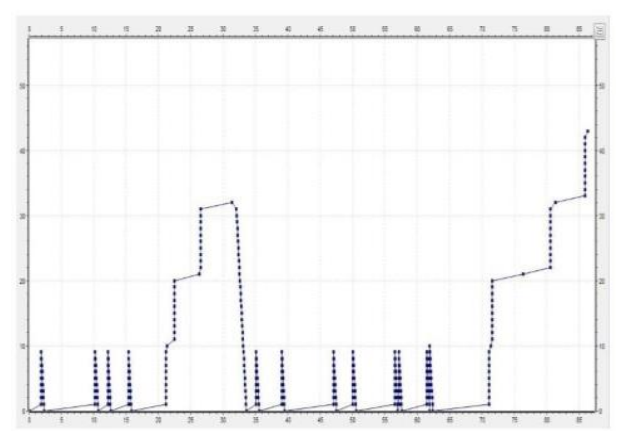

Figure 4. Queue time (in seconds) for the FIFO queue \& Queue time (in seconds) for the LIFO queue

For the outcomes part of the reproduction, we are for the most part keen on the lining time for every situation, and how this change with the position of the RSUs. For this, we extricated the information we recorded, from the vector documents: Maximum Queuing Time (MaxQt), Minimum Queuing Time (MinQt) and Average Queuing Time (AvgQt).

Table 2. Output results for various scenarios

\begin{tabular}{|c|c|c|}
\hline & FIFO & LIFO \\
\hline MaxQt & $489 \mathrm{~ms}$ & $760 \mathrm{~ms}$ \\
\hline AvgQt & $280 \mathrm{~ms}$ & $262 \mathrm{~ms}$ \\
\hline MinQt & $\mathrm{ms}$ & $39 \mathrm{~s}$ \\
\hline
\end{tabular}

\section{Conclusion}

We proposed a plan for distinguishing the rapid automobile. Our plan makes use of position of the vehicle to become aware of the short car. Every automobile offers its role and timing statistics to the RSUs and RSUs ship the information to the focal server. The relevant Server methods the everyday velocity of the automobile, clog and vehicle number using timing and function information gotten from the RSUs. on the point when an automobile is found to satisfy with a mischance, the important Server communicates these statistics to all RSUs in its range and for this reason the messages could be communicated to unique OBUs inner that location. At the point, when the vehicle once more comes into the scope of an RSU, it educates the automobile to take preoccupation.

\section{References}

[1] Lusheng Miao, "Performance evaluation of IEEE802.11p MAC protocol in VANETs safety application," IEEE, pp.1681-1685, (2013) DOI: 10.1109/WCNC.2013.6554813

[2] Qixiang Pang, "A rate adaptation algorithm for IEEE 802.11 WLANs Based on MAC-Layer loss differentiation,” IEEE, pp.709-716, (2005) DOI: 10.1109/ICBN.2005.1589671

[3] Seema.D and Sujata.Terdal, "Position based forwarding routing in real time city VANET," International Journal of Advanced Research in Computer Engineering \& Technology, pp.2580-2584, (2013)

[4] Vimmi A Gajbhiye, "Study of efficient routing protocols for international VANET," Journal of Scientific Engineering Research, vol.4, no.3, pp.1-9, (2013) 
[5] Theodore L. Willke, "A survey of inter-vehicle communication protocols and their applications," IEEE Communications Surveys \& Tutorial, vol.11, no.2, pp.3-19, (2009) DOI: 10.1109/SURV.2009.090202

[6] A,Katherine, "Vehicle-to-vehicle communication using RFID along with GPS and WAP," International Journal for computer science and mobile computing, vol.3, no.4, pp.21-27, (2014)

[7] Frank Kargl, "Secure vehicular communication systems: Implementation performance and research challenges," IEEE Communication Magazine, vol.46, no.11, pp.110-117, (2010) DOI: 10.1109/mcom.2008.4689253

[8] Pranav Kumar Singh, "Comparative study of radio propagation and mobility models in vehicular adhoc network," International Journal of computer Applications, vol.16, no.8, pp.37-42, February, (2011)

[9] Mihail.L.Sichittu, "Inter-vehicle communication systems: A survey," IEEE communication Survey, vol.10, pp.85-105, (2008) DOI: 10.1109/comst.2008.4564481

[10] Nellore Harika, Muthyala Vamsilatha, Nakka Thirupathi Rao, Debnath Bhattacharyya, and Tai-hoon Kim., "Performance analysis and implementation of traffic monitoring system using wireless sensor network for reducing blockage in Traffic on Indian City Roads," Journal of Statistical Computing and Algorithm, vol.1, no.1, Dec, GVPress, pp:15-26, (2017)

[11] Sarita Singh Bhadauria and Deepika Jaiswal., "Performance analysis of traffic type and routing protocols in VANET for city scenario," International Journal of Urban Design for Ubiquitous Computing, vol.4, no.1, Mar, GVPress, pp:1-12, (2016)

[12] Vasanth Ragu, Saraswathi Sivamani, Myungbae Lee, Hyunwook Cho4, Yongyun Cho, Jangwoo Park, and Changsun Shin, "Analysis of traffic congestion and identification of peak hour for intelligent transportation services based on IoT environments," International Journal of Smart Home vol.11, no.11, Nov, GVPress, pp:2532, (2017)

[13] Kiarash Ghasemlou, Metin Mutlu Aydın, and Mehmet Sinan Yıldırım, "Comparison of delay time models for over-saturated traffic flow conditions at signalized intersections," International Journal of Advanced Science and Technology, SERSC Australia, ISSN: 2005-4238 (Print), 2207-6360 (Online), vol.84, November, pp.9-18, (2015) 